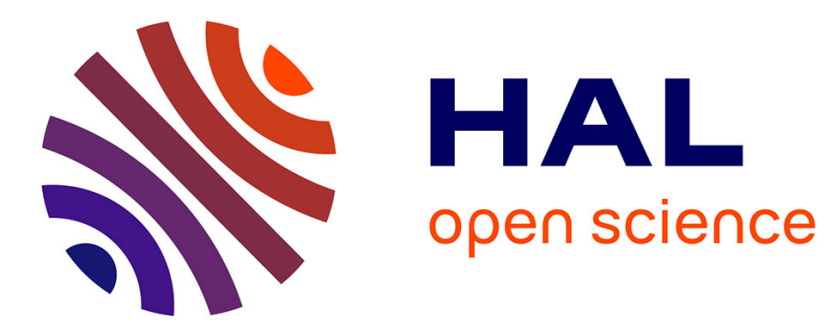

\title{
Advanced microstructure products by synchrotron radiation lithography
}

\author{
H. Lehr, W. Ehrfeld
}

\section{To cite this version:}

H. Lehr, W. Ehrfeld. Advanced microstructure products by synchrotron radiation lithography. Journal de Physique IV Proceedings, 1994, 04 (C9), pp.C9-229-C9-236. 10.1051/jp4:1994941 . jpa-00253502

\section{HAL Id: jpa-00253502 https://hal.science/jpa-00253502}

Submitted on 1 Jan 1994

HAL is a multi-disciplinary open access archive for the deposit and dissemination of scientific research documents, whether they are published or not. The documents may come from teaching and research institutions in France or abroad, or from public or private research centers.
L'archive ouverte pluridisciplinaire HAL, est destinée au dépôt et à la diffusion de documents scientifiques de niveau recherche, publiés ou non, émanant des établissements d'enseignement et de recherche français ou étrangers, des laboratoires publics ou privés. 


\title{
Advanced microstructure products by synchrotron radiation lithography
}

H. Lehr and W. Ehrfeld

IMM Institut für Mikrotechnik GmbH, Carl-Zeiss-Strasse 18-20, 55129 Mainz, Germany

\begin{abstract}
Deep X-ray lithography using synchrotron radiation with a characteristic wavelength of about $1-3 \AA$ is a powerful tool to generate threedimensional microstructures in thick layers of polymeric resists. Resist reliefs produced in this way are used in the framework of the so called LIGA process, as precise templates to generate microdevices from metals, plastics, ceramic materials, and glasses. A wide variety of applications exist for such microdevices in nearly all fields of modern technologies. Accordingly, development activities in LIGA microfabrication have been started worldwide. In the past few years materials and processes applied in deep $\mathrm{X}$-ray lithography have been improved considerably. This includes the development of a new positive tone polymer resist and a new negative tone resist based on chemical amplification, thereby exceeding the sensitivity of poly(methylmethacrylate), the most often utilized resist material so far. By means of beryllium and diamond membranes ultraprecise X-ray masks have been realized. Advanced equipment has been developed which facilitates a good overlay accuracy for generating threedimensional microstructures by multiple irradiation processes. In addition, the subsequent replication steps of the LIGA process as well as the corresponding materials have been further improved and first LIGA devices are produced commercially.
\end{abstract}

\section{Introduction}

In the last two decades powerful tools have been developed for fabricating microdevices. Techniques like wet-chemical anisotropic etching of single-crystalline silicon, or dry-etching processes by means of low-pressure plasma or ion beams mainly evolved from microelectronics, and are now successfully used in new disciplines like micromechanics and microoptics $(1,2)$.

A completely new approach is given with the LIGA technique. Due to the large variety of materials to be chosen from, qualities like optical transparency, mechanical stiffness and hardness, electrical conductivity and magnetic properties required for the specific functional behaviour of the microdevice may be optimized (3). This opens completely new opportunities for the realization of micromechanical, microfluidic and microoptical elements since important components like bearings, coils, microvalves, lenses, waveguides, channels and magnetic structures may now be realized by utilizing appropriate materials. As a consequence hybrid systems e. g. "milli" rotational motors, linear motors, micropumps, photonic systems and various analytical devices assembled with high precision LIGA components, became extremely attractive. 
The abbreviation LIGA originates from the German expressions for the major steps involved in this microfabrication technique: lithography, electroforming and moulding (German: Lithographie, Galvanoformung and Abformung). Structurization of a radiation-sensitive polymer is the first step in the LIGA process. This can be achieved by utilizing a laser, an electron or ion beam, as well as by opticalor X-ray lithography, the latter of which has been shown to give the best results. In this case an absorber pattern is transferred into an X-ray sensitive polymer (see fig. 1). In the subsequent development step the exposed or nonexposed regions of the resist are removed depending on the type of the resist. After the development process the narrow gaps of the resist relief are filled with a metal or metal alloy by electroplating, resulting in an extremely accurate metal structure, complementary to the original polymer structure (see fig 1). The metal form may be used for replication processes like moulding, embossing or further electroforming. It is the last step in the sequence of the LIGA process which offers the unique feature of inexpensive microstructure replication, with the potential of mass fabrication in industrial applications.

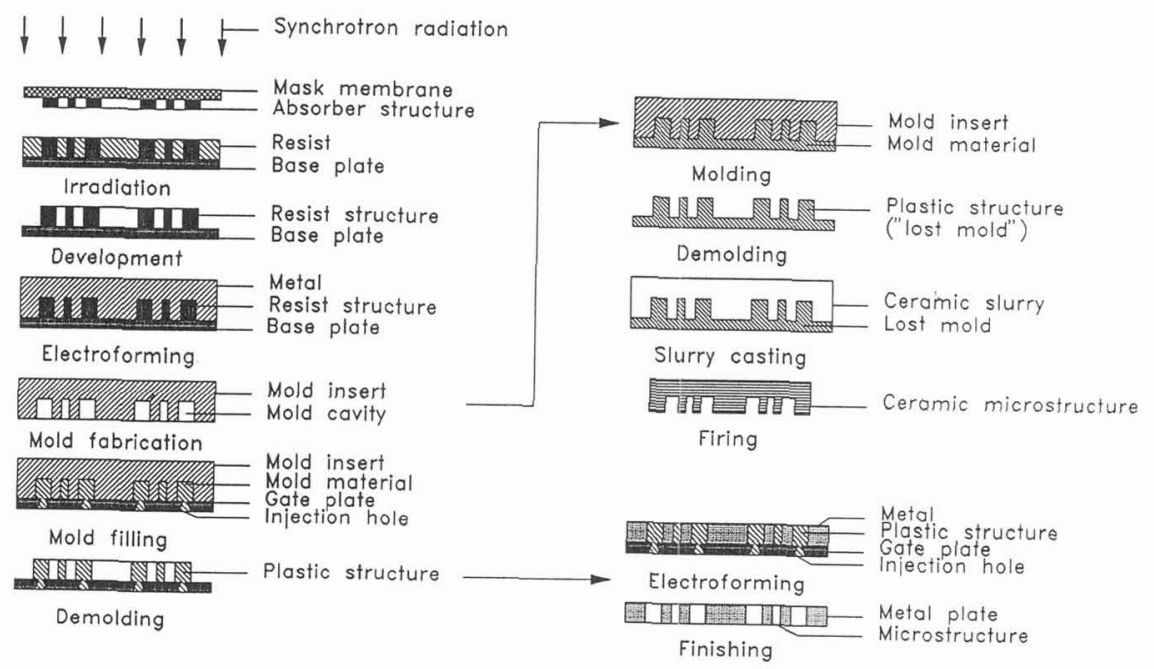

Fig. 1:Typical sequence for the production of microstructures using the LIGA process. Different fabrication steps may be chosen after deep X-ray lithography and resist development.

\section{Deep Lithography}

\subsection{Overview}

In advanced microelectronics the typical resist height is in the range of one micron with a structural width of half a micron. Accordingly, the so called aspect ratio, the quotient of the resist height and the structural width is typically in the order of two. However, micromechanical and microoptical elements, which are expected to be threedimensional in shape require aspect ratios ten to a hundred times larger. Therefore, "planar" lithography must be substituted by deep lithography with extremely high aspect ratios.

A typical arrangement for the performance of deep X-ray lithography at a synchrotron or storage ring is shown in fig. 2. The beamline, an evacuated tube which includes valves, a delay line and thin membranes to protect the vacuum system of the storage ring, guides the radiation to the lithography sta- 
tion at a typical distance of about $10-20 \mathrm{~m}$ from the source point. A filter system in front of the lithography station allows the smooth removal of the low energy radiation part, whereas absorption edges of selected materials may be used to suppress the extremely hard X-ray content of the spectrum.

Shadow printing is performed by use of an X-ray mask, a thin X-ray transparent membrane with an absorbing pattern which is transferred into a thick radiation sensitive polymer on a substrate. Only part of the mask and the substrate will be irradiated due to the small vertical opening angle of the radiation. A homogeneous dose distribution is usually achieved by scanning the assembly mask-substrate periodically in the vertical direction. For a high precision pattern transfer process the periodic scanning of the assembly mask-substrate in a so called X-ray scanner must be performed with high accuracy. Any varying tilt during the scan results in irradiation of the shadow region below the absorber and a corresponding loss of accuracy. Easy handling and a high precision in the mounting of mask and substrate as well as a complete control of all parameters in the irradiation process are further prerequisits for such an apparatus.
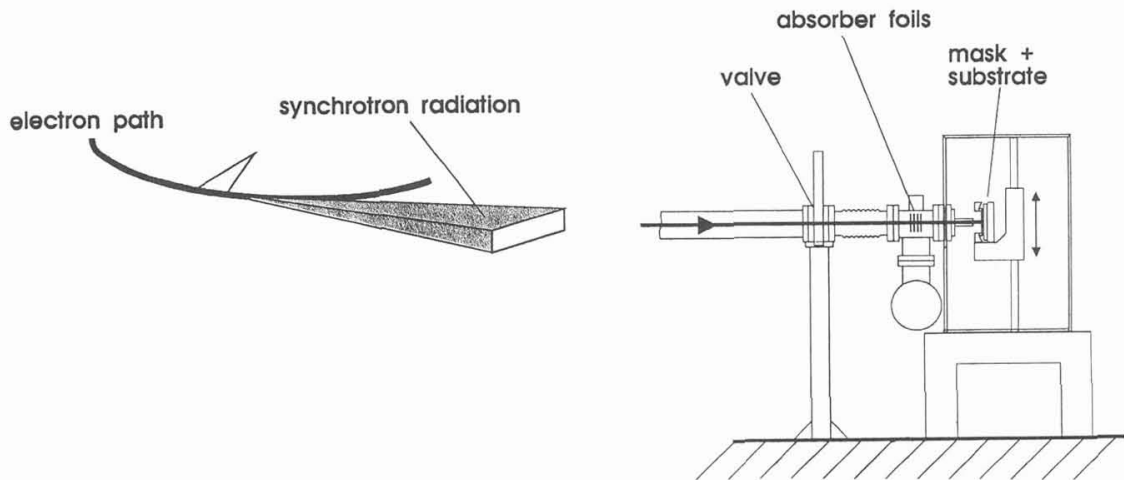

Fig. 2: Electron path in bending magnet, radiation fan and X-ray scanner for deep X-ray lithography.

The simple shadow printing process results in patterns with walls perpendicular to the substrate. However, there are a large variety of microstructures which e. g. require inclined walls or even microstructurization in several layers. A fixed slope of the mask-substrate assembly, rotary motions around the beam axis during the irradiation and an extremely precise relative mounting of mask and substrate, controlled by means of an alignment system, are some of the features of an advanced scanner system which has been developed by IMM and Jenoptik GmbH. It allows for variable shaping of the microstructures as a function of height, either stepwise due to multiple irradiation processes utilizing different masks, or continuously due to the tilt of the mask-substrate assembly. Alignment marks are necessary both on the mask and the substrate for the multi layer technique.

\subsection{X-Ray Masks}

An X-ray mask typically consists of a stable membrane, which is highly transparent for X-rays and an absorbing structure with a thickness of up to $20 \mu \mathrm{m}$ for deep lithography to keep the accumulated dose in the shadow region well below a certain threshold in the polymer resist in order to prevent unwanted erosion after irradiation. High contrast is achieved by utilizing membrane materials with low atomic charge, e. g. Be, C (diamond), polyimide, SiC, whereas the absorber deposited onto the membrane consists of high $\mathrm{Z}$ material, typically gold.

Due to the absorption edge of Si at around $7 \AA$ and the corresponding high absorption coefficient in the wavelength region 2 - $5 \AA$, Si and Si-compounds have serious drawbacks as membrane materials for deep X-ray masks. Ti is still utilized in certain cases. However, its relatively high absorption which 
limits the membrane thickness and therefore the heat conduction during the exposure, as well as its absorption edge at around $2.5 \AA$ certainly rule out this material. Be and C (diamond) have much lower absorption coefficients, which allow the formation of relatively thick $(0.5 \mathrm{~mm}$ in case of $\mathrm{Be})$ and stable membranes, an important issue with respect to possible stress and deformations occurring due to the deposition of the absorber onto the membrane and the temperature rise in the irradiation process. Besides X-ray transparency a number of further material properties have to be regarded for the mask membrane. These are e. g. Young's modulus, optical transparency, surface quality, chemical and irradiation stability, non-toxicity as well as production costs and process compatibility. In view of all these requirements, diamond membranes will be the best choice for the long run.

As absorber materials Au, W, Ta or Pt can be used. Since electroplating is the preferred process for generating the absorber pattern, $\mathrm{Au}$ is the optimum material. It is only if high temperature processes are required in the mask fabrication, that some problems are to be expected in the combination of $\mathrm{Au}$ with the preferred diamond membrane. This is due to the different thermal expansion coefficients of the two.

\subsection{X-ray Resist Materials}

Poly(methylmethacrylate) (PMMA) contiues to be used as a standard resist material for deep X-ray lithography. It is polymerized, starting from a PMMA resin, onto an electrically conducting substrate. The exposed areas mainly consist of low molecular weight PMMA, which can be selectively removed in an appropriate solvent (developer). A unique feature of PMMA is its extraordinarily high resolving power and the extremely low roughness of the resist structure walls. Furthermore, it can be used directly for the X-ray lithographical fabrication of e. g. microoptical components or mechanical filters with high precision pore sizes. The resist structures fabricated by means of deep X-ray lithography with PMMA exhibit only very small lateral structure deviations in the submicron range, even for structures several hundred microns high.

Adhesion of the resist on the substrate forms another important issue. In addition to an oxidized titanium substrate, silane with a double bond polymer group is admixed to the PMMA resin, resulting in a siloxane bond to the metallic surface of the substrate (3). New developments in this area have been undertaken at IMM using self-assembling layers. By this means a monolayer is generated on the substrate, which allows for a covalent bond between the resist and the substrate.

The main disadvantages of PMMA are its relatively low X-ray sensitivity and its susceptibility to stress corrosion. Current R\&D work in the field of resist development for deep X-ray lithography, performed by BASF company, has lead to a new resist material based on poly-(lactide-co-glycolide). Its $\mathrm{X}$-ray sensitivity is higher by a factor of two compared to that of PMMA and no stress corrosion occurs. The Max-Planck Institute for Polymer Research and the IMM are also working in this area, and have succeeded in developing a highly sensitive negative resist for deep X-ray lithography which is based on Novolak resins. Here the irradiated parts will be maintained contrary to positive tone resists. Since the principle of chemical amplification has been applied irradiation time is reduced, at least by a factor of 20 compared to PMMA. This resist material is therefore highly interesting for the direct fabrication of microstructures utilizing deep lithography which may form the final product in certain cases.

\subsection{Irradiation process}

Precise pattern transfer requires extremely parallel light, which will be obtained due to the use of synchrotron radiation. Fresnel diffraction is reduced due to the suppression of the long wavelength part of the broad synchrotron radiation spectrum. This is achieved by the installation of vacuum windows in the 
beamline, by appropriate filters and the absorption of synchrotron radiation in the mask membrane. The effect of absorbers on the power spectrum from the synchrotron radiation source DCI in Paris (positron energy $E=1.85 \mathrm{GeV}$, bending field $\mathrm{B}=1.6 \mathrm{~T}$, circulating positron current $\mathrm{I}=300 \mathrm{~mA}$ ) is shown in fig. 3 (full drawn line: angle integrated spectral distribution). The dashed curve in fig 3 represents the spectrum behind Be absorbers with a total thickness of $1 \mathrm{~mm}$ (vacuum window: $500 \mu \mathrm{m}$, mask membrane $500 \mu \mathrm{m}$ ) as it will appear on the surface of the resist in a typical lithography process. The absorber obviously shifts the maximum of the spectrum to shorter wavelengths and reduces the intensity. The lower curve represents the power spectrum at the bottom of the resist, i. e. on the surface of the substrate, due to a PMMA resist thickness of $500 \mu \mathrm{m}$.

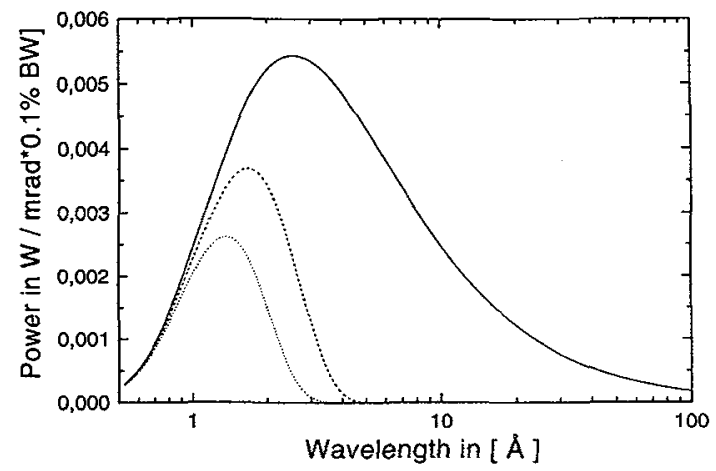

Fig. 3: Pure radiation spectrum of DCI, as well as after various absorbers.

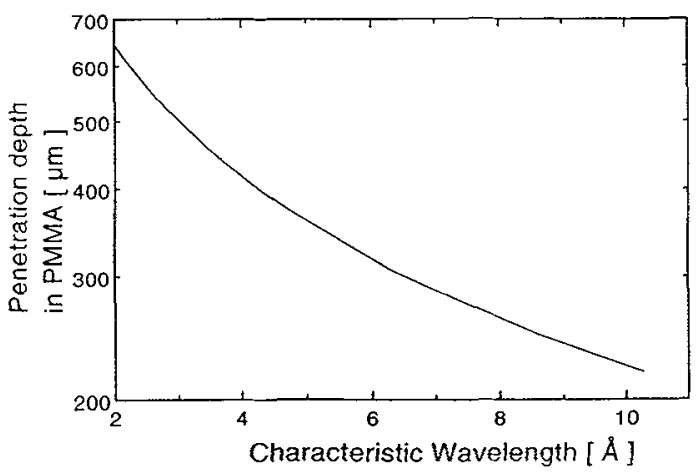

Fig. 4: Penetration depth in PMMA as a function of the characteristic wavelength.

Using PMMA as X-ray resist material a radiation dose of preferably $D D=4 \mathrm{~kJ} / \mathrm{cm}^{3}$ is chosen in depth, directly on the substrate. On the surface of the resist a maximum dose of DS $=20 \mathrm{~kJ} / \mathrm{cm}^{3}$ should not be exceeded. Higher doses result in the formation of cracks and bubbles. The X-ray penetration depth in PMMA as a function of the characteristic wavelength with a dose relation of DS/DD $=5$ is shown in fig. 4. A depth of more than $500 \mu \mathrm{m}$ in PMMA and higher aspect ratios may be obtained by choosing the critical wavelength smaller than $\lambda c=3 \AA$. The results presented in fig. 4 are again based on a Be absorber with a thickness of $1 \mathrm{~mm}$. Higher penetration depths may be achieved by using thicker absorbers.

\subsection{Advanced lithography processes}

Up to now an X-ray beam impinging perpendicular onto the mask and the resist/substrate system has been considered. By simply inclining both with respect to the beam axis resist structures with oblique angles will be obtained after the development process, or even dovetail structures utilizing two different irradiation angles like in fig. 5. A simple example for such a structure is given in fig. 6 showing grooves for the embedding of optical fibres and a wall with an inclination of 45 degrees in front of one fibre. A light beam passing the fibre may easily be deflected out of plane.

Oblique irradiation is one possible way to perform a continuous vertical structurization in the lithography process. Another method is multiple exposure in order to achieve discontinuous structures like it is shown in fig. 7. In the first irradiation step a microstructure pattern and alignment marks are transferred into the resist. After dissolving the irradiated parts in an electroforming process the holes in the resist will be filled with metal in an electroforming process. Then a second resist layer is deposited and a further exposure is carried out using a mask with a different pattern which has been aligned with 
respect to the marks of the first structure. An example for such structures representing electroformed gear wheels is shown in fig. 8 .

High precision mask alignment, inclination of the resist/substrate and rotation around the $\mathrm{X}$-ray beam axis are additional features which are included in the scanner system developed by IMM/Jenoptik, thereby enhancing the "tools" for three-dimensional structurization by lithographical methods considerably.

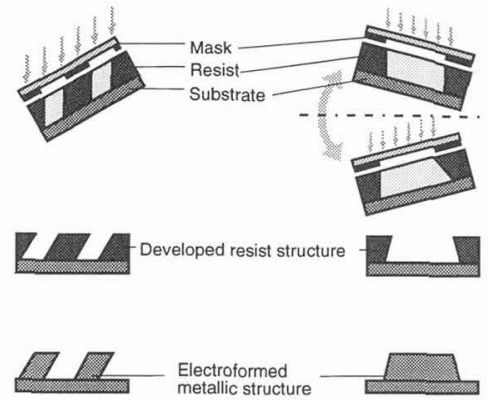

Fig. 5: Oblique irradiation steps.

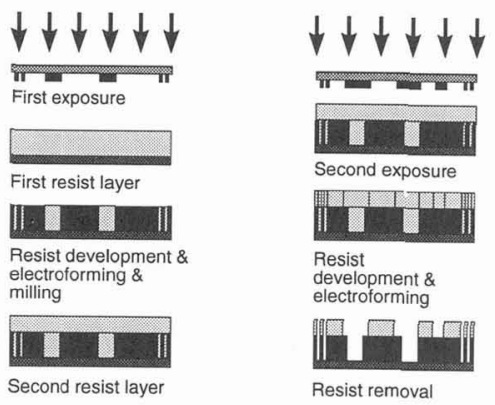

Fig. 7:Production steps for multiple exposure.

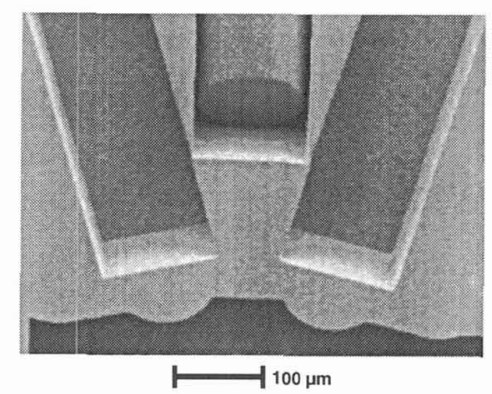

Fig. 6: Inclined wall for out of plane reflection.

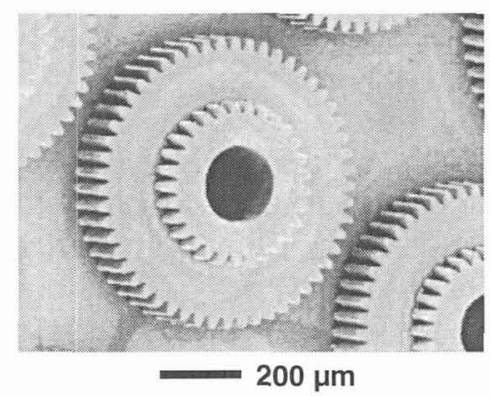

Fig. 8: Two stage nickel gear wheel.

\subsection{Microelectroforming}

Electroforming is the second major production step in the sequence of the LIGA technique. After irradiation and development the resist gaps may be filled with a large variety of metals and metal alloys in an electrochemical deposition process.

Electroforming constitutes an industrial manufacturing process often utilized in hybrid microelectronics, which nonetheless differs in some details from the procedure needed for the homogeneous and defect-free precipitation of a metal in the narrow and deep gaps of a LIGA structure. Wetting agents are an unavoidable ingredient in the microelectroforming process, since the complete wetting of the resist structures and the substrates is an absolutely necessary prerequisite for defect-free electroforming. The electrolytes have to be very pure and impurities due to dust, anodic mud, disintegration of the electrolyte or its additives have to be carefully avoided by filtration. Such impurities can change the material properties, cause defects and could possibly form pinning centers for hydrogen bubbles, which locally inhibit the metal deposition. 
A number of electroforming processes have been developed to date for application in the LIGA process. These include pure metals like $\mathrm{Ni}, \mathrm{Cu}, \mathrm{Au}, \mathrm{Ag}, \mathrm{Pd}, \mathrm{Fe}, \mathrm{Cr}$ and $\mathrm{Zn}$ as well as binary alloys like $\mathrm{Ni}-\mathrm{Co}$, $\mathrm{Ni}-\mathrm{P}, \mathrm{Ni}-\mathrm{Fe}, \mathrm{Ni}-\mathrm{B}, \mathrm{Cu}-\mathrm{Sn}$ and $\mathrm{Cu}-\mathrm{Zn}$. Furthermore, compound materials like Ni-PTFE, Ni-diamond and $\mathrm{Ni}$-TiN have been proven to be applicable. In addition, current experimental work deals with microelectroforming of $\mathrm{Al}$ and further metals, as well as metal alloys which can only be deposited from non-aqueous electrolytes. Another field for innovative applications has opened by the electrolytic deposition of intrinsically conducting polymers.

Material properties may be adjusted to the specific need of the final microstructure product within certain limits by varying parameters of the electroforming process like current density, temperature, ion concentration and variations of special additives like the above mentioned wetting agent. Adjustable material properties are e. g. Young's modulus, internal stress, surface roughness and electrical conductivity. Recent interests in magnetically driven microactuators fabricated by means of the LIGA method stimulated R\&D work for the improvement of magnetic parameters like magnetic anisotropy, coercivity, the saturation value of magnetization and the general form of the hysteresis loop (4).

As an example for the application of LIGA microstructures being used for the realization of electromagnetically driven actuators, a completely mounted "milli"motor is shown in fig. 9. An exploded schematic view (fig. 10) presents the different components of the assembly: stator, double sided coils, rotor and distance ring, made by use of the LIGA technique. Two conventionally fabricated micro-ball bearings and a housing tube with an outer diameter of $2 \mathrm{~mm}$ are further components. Such motors may be used in medical technology, miniaturized hard disk assemblies or drive systems for portable electronic equipment.

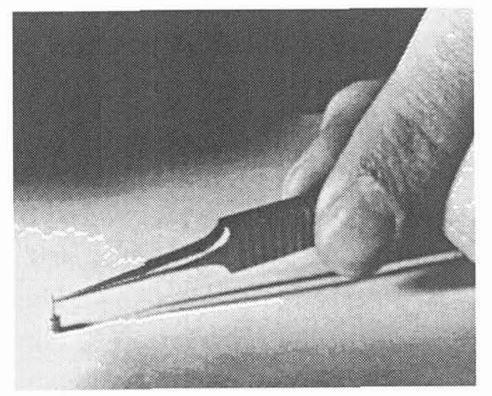

Fig. 9: Completely assembled "milli"motor.

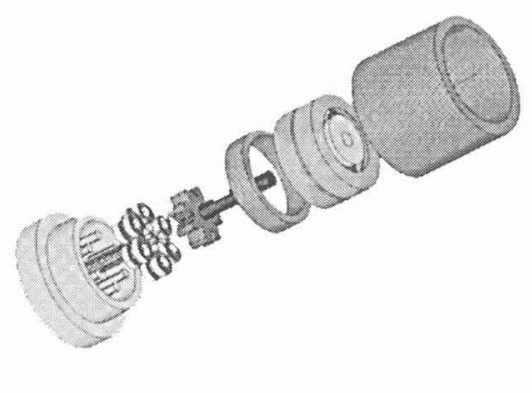

Fig. 10: Exploded view of "milli"motor.

\subsection{Moulding Processes}

For the fabrication of microstructures with extreme structural heights by means of micromoulding, reaction injection moulding or embossing, the roughness of the cavity walls of the mould insert is a decisive factor. In the typical process sequence for the production of mould inserts by deep X-ray lithography with a PMMA resist and the subsequent electroforming with nickel, an average roughness of less than $50 \mathrm{~nm}$ is achieved.

Standard injection moulding machines are suitable for the production of polymer structures by micromoulding. However, a procedure with variable temperature has to be used. At the beginning of the process the mould insert is kept near the melting temperature of the mould material and filled. The temperature is reduced afterwards until the mould material has reached sufficient strength, and 
separation of the mould from the mould insert can take place. Before filling, the mould insert cavities must be evacuated.

Appropriate materials to be used for micromoulding must have a low viscosity in the molten state. Many materials have been tested to date like poly(methylmethacrylate), polyoxymethylen, polyamide, polycarbonate, polystyrene and polyvinylidenefluoride as well as various co- and blockpolymers. In addition, casting resins based on epoxy, methacrylate, silicone and polyamide materials have been tested for micro reaction injection moulding.

In many cases, injection moulding can be replaced by hot embossing. Favourable short process times are achievable and even details in the order of $10 \mathrm{~nm}$ can be transferred from a nickel master into a polymeric material.

Ceramic microstructures have been produced by applying slip casting processes introducing a ceramic slurry into a complementary plastic microstructure which is removed in afiring process. Products based on alumina, zirconia and piezoelectric (PZT) materials have been developed in this way. Besides ceramic powders, preceramic polymers are highly promising. Microstructures may first be generated from these precursors by means of a moulding process and then by a firing process converted into ceramic microstructures. The surface roughness is below $100 \mathrm{~nm}$. Thus the material may even be applied for optical microstructures. Fuel injection nozzles, chemical microreactors and microfiltration membranes constitute further applications.

\subsection{Conclusion}

Development work for the LIGA process has been carried out or started in various European countries as well as in the USA and the Far East. At present LIGA is moving from the research phase to a commercial production process, driven by the market's demand and a huge application potential which is due to the inherent advantages of the LIGA products. To carry out all process steps with a high yield and accuracy is nevertheless a real challenge.

In order to speed up the development and introduction of the LIGA process, IMM has started an extensive technology transfer program and installed a research network which are both supported by the European Community. In addition, professional production equipment like the X-ray scanner and electroforming units as well as materials optimized for the LIGA process are meanwhile available. Thus the technological threshold and the financial risk to introduce the LIGA process have been reduced considerably. However, a successful commercial implementation must be accompanied by ongoing fundamental research in many disciplines. The synchrotron radiation centers are expected to play a dominant role in performing this task.

\section{Acknowledgement}

The authors wish to express their gratitude for the ongoing support of the LURE staff for performing deep X-ray lithography experiments at the positron storage ring DCI in Paris.

\section{References}

[1] K. E. Peterson, Proc. IEEE (1982), Vol. 70, pp. 420 - 457

[2] R. S. Muller, Sensors and Actuators (1990), Vol. A 21, pp. 1 - 8

[3] W. Ehrfeld, H. Lehr, Radiation Physics and Chemistry (1994), Pergamon Press, in press

[4] H. Lehr, W. Ehrfeld, M. Schmidt, E. Kallenbach and H. A. Tuan, Journ. of Micromech. and Microeng. 2 (1992), pp. 229 - 233 